\title{
CHARACTERIZATION OF THE FACILITY FOR ATMOSPIIERIC CORROSION TESTING (FACT) AT SANDIA
}

C. J. Greenholt, N. R. Sorensen,

G. A. Poulter, and T. R. Guilinger

Sandia National Laboratories

Albuquer(jue, New Mexico 87185

\begin{abstract}
The capability to perform atmospheric corrosion testing of materials and components now exists at Sandia resulting from the installation of a system called the Facility for Atmospheric Corrosion Testing (FACT). This report details the design, equipment, operation, maintenance, and future modifications of the system. It also presents some representative data acpuired from testing copper in environments generated by the FACT.
\end{abstract}




\section{Contents}

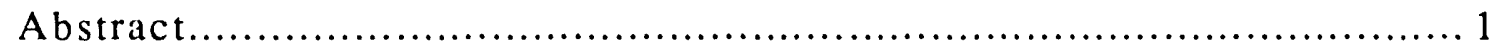

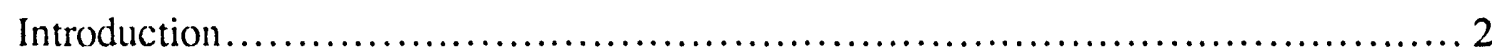

Initial Design Criteria ..................................................... 2

System Description ..................................................... 3

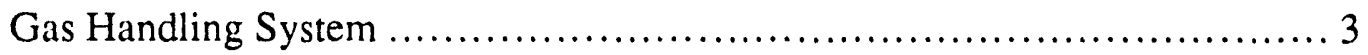

Temperature-Controlled Chamber............................................ 4

Gas Analysis................................................... 5

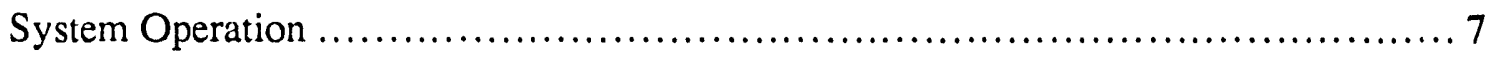

Maintenance............................................................. 8

Future Planned Modifications ............................................. 8

Results - Chamber Uniformity ........................................... 9

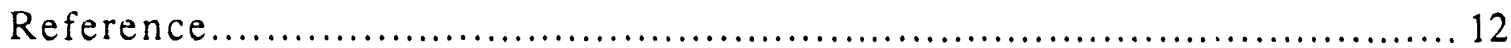




\section{CHARACTERIZATION OF THE FACILITY FOR ATMOSPHERIC CORROSION TESTING (FACT) AT SANDIA}

\section{Introduction}

Corrosion is the destructive attack of a metal by chemical or electrochemical reaction with its environment. Some very severe environments can cause total destruction of a material while other, more benign environments will only result in the formation of a thin film on the material's surface. Atmospheric corrosion is considered the latter of the two and can be defined as the reaction of a metal with atmospheric air, i.e. air containing low concentrations of contaminants such as sulfur dioxide, hydrogen sulfide, nitrogen dioxide, chlorine, and water vapor. Industrial air frequently contains these contaminants in concentrations ranging from parts per billion to parts per million [1].

Electrical contact failure is one problem resulting from atmospheric corrosion. The thin films formed by the reaction of environmental pollutants with the metal surfaces of contacts has long been recognized as a major threat to contact reliability because of their adverse effect on contact resistance. The composition of the films depends on the metal and the type and concentration of the contaminants present. In addition, at least one study has shown that there are synergistic effects of complex gas mixtures [1].

To investigate atmospheric corrosion in the laboratory, a system with well-defined capabilities is required. This report documents the design, construction, operation, maintenance, future plans, and also shows some representative sample data for a system constructed recently at Sandia. The system is known as the FACT (Facility for Atmospheric Corrosion Testing). The construction of the FACT has made available a facility for performing sophisticated atmospheric corrosion testing on a variety of materials and components.

\section{Initial Design Criteria}

The system should be capable of:

1. Accurately maintaining the individual concentrations of up to four contaminant gases at levels between 5 and $1000 \mathrm{ppb}$,

2. Controlling the relative humidity over the range from 30 to $95 \%$, 
3. Maintaining a constant sample temperature between $30^{\circ}$ and $70^{\circ} \mathrm{C}$, and

4. Maintaining a maximum constant flow rate equivalent to six reaction chamber volumetric exchanges per hour.

The following features are also desirable:

1. A reaction chamber volume of approximately one cubic foot,

2. The ability to see the samples under test without disturbing the test conditions,

3. Simplistic design that utilizes commercially available components that are inert to the test environment, and

4. Uncomplicated and low maintenance operation.

\section{System Description}

Figure 1 shows a schematic diagram of the complete system. The system can be divided further into three subsystems: the gas handling system, the temperature-controlled reaction chamber, and the gas analysis system. We will describe the system from beginning to end in the direction that the gas flows, i.e. beginning at the inlet to the gas handling system, continuing through the reaction chamber, and then concluding at the analyzers.

\section{Gas Handling System}

Compressed air (75 to 150 psi) is used as the carrier for the contaminant gases and the water vapor. Before the compressed air can be used, it must be dried, purified, and its pressure must be regulated. The air first contacts a combination coalescent filter and pressure regulator to remove oil and water and reduce the air pressure. The air then passes through a desiccant-type regenerative dryer for final drying. This dry air then passes through a particulate filter to remove any desiccant particles, then passes to a carbon tilter to purify the air. Finally, a second regulator further reduces the pressure to approximately 15 psi for inlet to the mass flow controllers. This process yields a carrier gas supply (up to 4.5 liters per minute with the inlet to the regenerative dryer set at 7.5 psi) of pure. (iry air at 1.5 psi.

As seen in Figure 2, using two sets of Sierra Instruments gas malss flow controllers, labeled P1 through P4 and R1 through R4, and Teflon back pressure/vent valves, desired concentrations of contaminant gases are introduced into the dry air stream. The first set of mass flow controllers is used to maintain a constant flow of purified air across the permeation tubes. This produces an air stream with known concentrations of contaminants. The second set of controllers is used to establish the quantity of contaminant gas that is introduced into 
the test chamber, with any excess flow being vented. The back pressure valves serve two functions: they provide a means to adjust the back pressure on each " $R$ " valve, and they allow any excess flow from the "P" controllers that does not pass through the corresponding " $R$ " controllers to be diverted and vented. Permeation tubes manufactured by GC Industries are the contaminant gas sources. Each of the contaminant gas streams flow into a gas mixing chamber and then into the oven, via a $1 / 8$ inch diameter Teflon tube, where they are mixed with air and water vapor immediately before entering the reaction chamber.

The FACT uses a heated, one liter, round bottom flask containing a bubbler submersed in deionized water to provide a source of water vapor to control relative humidity (RH). A portion of the FACT's carrier gas is diverted through this saturator producing essentially 10()$\%$ RH air. The saturated air is then mixed with a stream of dry air to produce the desired RH. The saturator is kept at a constant temperature by using a digital set-point temperature controller in conjunction with a variable transformer to supply the required power to a heating mantle. A type K (chromel-alumel) thermocouple, located in the satturator, provides the feedback to the controller, where the water temperature is also displatyed. This moist air stream then passes into the oven through a $1 / 4$ inch Teflon line.

\section{Temperature-Controlled Chamber}

Since testing is to be conducted at accurately controlled temperatures above ambiem (.3) to 7() ) , an oven is required. We chose to use an incubattor since the temperattures atre low and it is desirable to view the samples without disturbing the test conditions, . The incubator has glass doors within outer doors which permit viewing the simples. The internal dimensions are approximately $30 \times 24 \times 18$ inches.

The humidified air stream enters the oven via a $1 / 4$ inch heilted Teflon tube. Once inside the oven, the humidified air stream passes through a condensing vessel which removes excess moisture if the dew point of the humid air stream exceeds the temperature of the oven. The contaminant air stream enters the oven via a $1 / 8$ inch Teflon tube and combines with the humid air downstream from the condenser. This prevents dissolution of the contaminants by the condensed water prior to entering the reaction chamber.

The reaction chamber, locited inside the incubator, is a standard vacuum ilesiccittor with a glass deor for ealse in viewing the samples. The chamber measures approximately 12 inches on a side, thus providing a working velume of one cubic foct. The mixed contaminant gals stream and humidified air enter the bottom of the chamber through a $1 / 4$ inch diameter Teflon distribution tube that has been formed into a circle with the end pluggered. Small holes, about $1 / 16$ inch in diameter, perforate the circumference to provide a uniform distribution of the inlet gasses. Placed within the circular inlet distribution tube is an 
instrument-type cooling fan used to circulate the gases to minimize concentration gradients. There is also an Inconel-sheathed Type $\mathrm{K}$ (chromel-alumel) thermocouple inside the reaction chamber to measure the actual internal chamber temperature. The gas exits the top of the reaction chamber through $1 / 4$ inch Teflon tubing which then exits the incubator.

\section{Gas Analysis}

Once the gas line exits the oven it is heat traced again to prevent condensation. The moist gas stream passes through a heated housing containing a dew point hygrometer probe that measures the dew point of the stream. To analyze the gas stream to determine the concentration of the contaminants, all the moisture must first be removed. To accomplish this, the gases pass through a Perma-Pure dryer to remove the moisture before analysis. Perma-Pure dryers use a hygroscopic, ion exchange membrane to selectively remove water vapor from mixed gas streams. This membrane is an extrudable desiccant in tubular form. Severai of these tubes are bundled with a common header and sealed within a tubular shell. Effluent gas passes through these tubes while a countercurrent of dry air, provided by the regenerative dryer, flows over the tubes. The differences in water vapor pressure between the tubes and the shell provide the driving force to remove water without affecting the concentration of the other gas contaminants. The gas exiting the dryer encounters a threewaly stopcock. The gas stream can either be analyzed or vented.

Three analyzers are presently in use. A Columbia Scientific Instruments (CSI) Model 1600 Nitrogen Oxide Analyzer is used to measure the nitrogen dioxide concentration. The model 1600 utilizes the chemiluminescent method of analysis. The chemiluminescent method is based on the principle that nitric oxide (NO) reacts with ozone $\left(\mathrm{O}_{3}\right)$ to produce nitrogen dioxide $\left(\mathrm{NO}_{2}\right)$ in an electronically excited state $\left(\mathrm{NO}_{2}{ }^{*}\right)$, and oxygen $\left(\mathrm{O}_{2}\right)$. As the excited $\mathrm{NO}_{2}$ goes back to the ground state a photon (hv) is emitted, with the intensity of this emission being proportional to the amount of $\mathrm{NO}$ present.

$$
\begin{gathered}
\mathrm{NO}+\mathrm{O}_{3} \rightarrow \mathrm{NO}_{2} *+\mathrm{O}_{2} \\
\mathrm{NO}_{2} * \rightarrow \mathrm{NO}_{2}+\mathrm{hv}
\end{gathered}
$$

The analyzer can measure $\mathrm{NO}, \mathrm{NO}_{2}$, or $\mathrm{NO}_{x}$. By using a converter, $\mathrm{NO}_{2}$ is reduced to NO. Gas samples entering the analyzer pass through a three-way valve (operating at approximately 480 cycles/hour) which alternately bypasses the converter to detect only NO or routes the gas through the converter to measure $\mathrm{NO}+\mathrm{NO}_{2}$, defined as $\mathrm{NO}_{\mathrm{X}} . \mathrm{NO}_{2}$ is then determined by electronically subtracting the $\mathrm{NO}$ signal from the $\mathrm{NO}_{\mathrm{X}}$ signal. The concentration is displayed on an analog meter, or can also be recorded on strip charts or other devices via voltage output jacks on the rear of the unit.

The sulfur concentration is measured using a CSI Model SA-285-E Sulfur Analyzer. 
The SA-285-E is a flame photometric detector based on the chemiluminescence of sulfur species produced in a hydrogen hyperventilated flame. Molecules containing sulfur are converted to an $\mathrm{S}_{2}$ species by the hydrogen flame. Hydrogen and hydroxyl radicals are produced by the flame and react with $\mathrm{S}_{2}$ to produce an excited state of $\mathrm{S}_{2}$ :

$$
\begin{aligned}
& . \mathrm{H}+. \mathrm{H}+\mathrm{S}_{2} \rightarrow \mathrm{S}_{2}^{*}+\mathrm{H}_{2} \\
& . \mathrm{OH}+. \mathrm{H}+\mathrm{S}_{2} \rightarrow \mathrm{S}_{2}{ }^{*}+\mathrm{H}_{2} \mathrm{O}
\end{aligned}
$$

As the $\mathrm{S}_{2}$ * reverts back to a lower energy state, photons are emitted, with the intensity of this emission being proportional to the square of the sulfur present. This is a total sulfur analyzer, and therefore, if both hydrogen sulfide and sulfur dioxide are used simultaneously in the system, the detected sulfur concentration will be comprised of the contribution of each sulfur species. To determine the contribution of each species, the hydrogen sulfide is then scrubbed from the gas stream with a filter attached to the back side of the analyzer. The resulting sulfur signal is then attributable only to sulfur dioxide and the difference between this signal and the total sulfur signal determines the hydrogen sulfide concentration.

The chlorine concentration is measured using a Mast Model 724-5 Oxidant Monitor. This analyzer uses a chemical solution and a microcoulomb sensor to determine the chlorine concentration. The analyzer contains a microcoulomb sensor over which a thin film of sensing solution flows. The microcoulomb sensor is comprised of several turns of fine platinum wire serving as a cathode, and two turns of platinum wire as an anode. A gas stream is pumped through he sensor and any gaseous oxidants are then detected by the oxidation of potassium iodide contained in the sensing solution as follows: (Other oxidants such as $\mathrm{Cl}_{2}, \mathrm{Br}_{2}, \mathrm{~F}_{2}$, and $\mathrm{I}_{2}$ react similarly)

$$
\mathrm{O}_{3}+2 \mathrm{KI}+\mathrm{H}_{2} \mathrm{O} \rightarrow \mathrm{O}_{2}+\mathrm{I}_{2}+2 \mathrm{KOH}
$$

Hydrogen gas is evolved at the cathode producing the polarization current:

$$
2 \mathrm{e}^{-}+2 \mathrm{H}^{+} \rightarrow \mathrm{H}_{2}
$$

As free iodine is produced by the reaction with the oxidant $\left(\mathrm{O}_{3}\right)$, it immediately reacts with $\mathrm{H} 2$ as follows:

$$
\mathrm{H}_{2}+\mathrm{I}_{2} \rightarrow 2 \mathrm{HI}
$$

The removal of hydrogen from the cathode produces a repolarization current of two electrons to restore equilibrium. Each ozone molecule generates two electrons upon reacting on the sensor, and therefore, the total electron flow is proportional to the ozone (or oxidant) present. The output of the analyzer is this microampere current.

Different oxidants can affect the output of the chlorine analyzer. Sulfur dioxide interferes in a negative manner and reduces the oxidant-caused signal. Nitrogen dioxide will cause a $6-10 \%$ increase in the reading. There are two ways to deal with interferences from other gases: the interfering gases can either be removed (filtered, absorbed) prior to the 
analysis, or they can be added to the gas stream after the chlorine component has been analyzed and adjusted to the desired concentration. The former method allows for both the monitoring and control of the desired gas concentration while the second method can only be used to monitor the gas concentration. The FACT uses the second method where the oxidant monitor is first calibrated using a gas stream containing a known concentration of only $\mathrm{Cl}_{2}$, and then, prior to a test, the $\mathrm{Cl}_{2}$ concentration in the gas stream is adjusted until the desired concentration is detected at the oxidant monitor. The $\mathrm{Cl}_{2}$ flow is maintained at this value for the duration of a test. The other contaminant gases to be monitored and controlled are then allowed to enter the system.

No positive or negative pressure can be tolerated by any of the three analyzers. Therefore, it is imperative that the manifold, from which the three analyzers simple the gas, be at atmospheric pressure.

\section{System Operation}

We flow purified, dry air through permeation devices, and control the contaminam concentration by ratioing the flow rates between that flowing through the permeation devices (P1-P4 controllers in Fig. 2) and that flowing into the reaction chamber (R1-R4 controllers). We then analyze the effluent and adjust the flow through the second set of flow controllers (R1-R4) accordingly. This is continued until the effluent concentration reaches the desired value for each contaminant species. This ability to analyze during testing is lundamental to maintaining controlled contaminant concentrations.

The initial flows through the R1-R4 controllers can be calculated using formulats provided with each of the permeation devices. However, this flow will change as test coupons are placed in the system, because they begin reacting with the contaminants, thus lowering eftluent concentrations. Compensating for these changes involves changing the

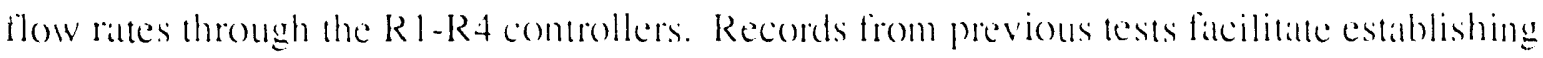
new initial tlow rates. It then becomes necessary to observe the concentration levels periodically, and o change flow rates accordingly. A smip cham reconder cam be used an continuously record the concentrations, particularly for the galses most affected.

The sattuator, operating at a temperature of approximately $38^{\circ} \mathrm{C}$ and a thow rate of 2800 standard cubic centimeters per minute (SCCM), uses approximaltely 125 cubic centimeters of water per day to produce a relative humidity of $70 \%$ at $38{ }^{\circ} \mathrm{C}$ in the reaction chamber. The salturator can easily operate for several days without refilling. However, daily refilling is 
recommended to maintain a more constant relative humidity. To increase the relative humidity of the system, the temperature of the saturator is increased and to decrease the relative humidity, the saturator temperature is decreased. The gas lines carrying humidified air downstream of the saturator must be heat traced to prevent condensation from occurring. Routine calibration of the analyzers is mandatory to assure accurate contaminant concentration levels. Calibration involves first introducing pure, dry air (referred to as zero) air in the operating manuals for the analyzers) and adjusting the analyzer zero knob on the front of the analyzer to read a zero concentration. Next, zero air is passed over a permeation device to generate a known contaminant concentration (determined from the permeation tube calibration sheet) and then the span knob is adjusted to give an analyzer reading equal to the calculated concentration. These two steps are then repeated until both zero and span knob settings are repeatable.

\section{Maintenance}

Several components of the FACT require periodic maintenance. All analyzers require periodic maintenance as does the carrier gas drying and purification eyuipment. The hydrogen cylinder supplying gas to the $\mathrm{SO}_{2}$ analyzer is depleted about every three weeks and must be replaced, and the drying/purification filters need replacement every six months. Infrequently, the mass flow controllers will lose there ability to control accurately, and must be sent back to the factory for repair and recalibration.

The permeation devices (GC Industries, Inc.) have a useful life of one year, except for chlorine with a six month life. The useful life is independent of whether the tube is being used or stored.

\section{Future Planned Modifications}

To improve the contaminant concentration stability in the system and reduce optrattor time and error, we plan to add two calpabilities to the system. The first is the addition of a computer, to monitor and record the signal from the analyzers and to provide and record a set point for the gas flow controllers. This continuous monitoring of the analyzers and controllers will assure a constant concentration of the contaminants in the chamber, and it will provide a record of the guantity of contaminants used at any time during the test.

The second addition is the installation of a level-maintaining atutomatic fill device for 
the saturator. Since small quantities of water will be added to maintain a set volume, the temperature of the saturator will remain more constant. A constantly maintained liquid level will also allow an increase in efficiency and consistency. Due to the tapered shape of the flask used for the saturator, the exposed surface area of water to the incoming air flow is minimal. As the water level falls, an increasing area is exposed thus increasing the efficiency. With the installation of a level-maintaining device, we can maintain a level nearer the bottom of the flask where the surface area is larger and the operating efficiency is higher. The constant level will result in a more stable relative humidity.

\section{Results - Chamber Uniformity}

The reaction chamber conditions we have tested are $100 \mathrm{ppb}$ hydrogen sulfide, $100 \mathrm{ppb}$ nitrogen dioxide, and $60-70 \%$ relative humidity at a chamber temperature of $38 \mathrm{C}$. We conducted tests, using copper samples, to evaluate the uniformity of contaminant concentration in the reaction chamber. The effects of cleaning techniques, i.e. comparing copper samples abraded with 600 grit $\mathrm{SiC}$ with those chemically cleaned in acid, have also been investigated. The effects of various inhibitors on copper have also been examined. We detail below the results of the chamber uniformity testing.

We performed exposures of $\mathrm{Cu}$ coupons to determine the effect of location in the chamber on the corrosion rate as measured by weight gain of the coupon. We prepared the coupons for exposure by degreasing them in acetone followed by hand polishing with 600 grit $\mathrm{SiC}$ paper to a uniform surface appearance. We then exposed the weighed samples for approximately one week to an atmosphere consisting of $\mathrm{H}_{2} \mathrm{~S}$ and $\mathrm{NO}_{2}$ at $35 \mathrm{C}$ and $60 \%$ relative humidity. Intermediate weights were recorded at approximately 1,2, and 4 day intervals with final weights recorded at the end of the exposure.

In the first test, five samples were placed on one rack in the top of the chamber ( 8 inches above the fan outlet). Figure 3 shows the weight changes of the samples with exposure time while Table 1 summarizes the weight change data. Relative standard deviations were less than 7\% for this sample group at every weighing and did not change systematically with exposure time. We concluded that sample position near the center of the uppermost rack does not significantly affect the sample corrosion rate.

For the second test, we placed three racks of samples, one each at the top ( 8 inches above the fan outlet), middle ( 5 inches above the fan outlet), and bottom ( 2 inches above the fan outlet) of the chamber. Four samples were distributed on each rack. Figure 4 shows the 
weight changes of the samples with exposure time. For clarity in this figure, only samples 3 , 9, and 11-12 are labeled. The weight change data for the remaining samples are grouped between samples 3 and 12. Table 2 summarizes the weight change data. As seen in Figure 4 and Table 2, uniform corrosion rates are exhibited on the top and middle racks, while the bottom rack has three samples $(9,11$, and 12$)$ with much higher corrosion rates. Relative standard deviations including the high samples ranged from 17 to $29 \%$. When these samples are excluded, the relative standard deviations were near $5 \%$ for all weighings with no systematic change with exposure time.

In the first two tests, the gas inlet tube was placed in a circle around the fan. This tube had $131 / 16$ inch diameter holes drilled at equal spacing around its 28 inch circumference ( 9 inch diameter). Because of the non-uniformity exhibited by the samples on the bottom rack in the previous uniformity test, we changed the configuration of the gas inlet tube for the next uniformity test to be a straight tube 4 inches long located directly under the fan inlet. This tube also had $131 / 16$ inch diameter holes drilled at equal spacing beneath the fan.

Figure 5 shows the weight changes of the samples with exposure time while Table 3 summarizes the weight change data for coupons exposed using the straight gas inlet tube. For clarity, the weight changes in Figure 5 do not have sample number labels on all curves. The weight change curves for samples 1, 4-7, and 12 all lie between samples 2 and 8 in this figure. Relative standard deviations, listed in Table 3 , show again that corrosion rates are much higher than average for samples on the bottom rack. When these samples are excluded, relative standard deviations are less than $12 \%$ for all weighings. However, since these standard deviations are higher than for the circular gas inlet tube, we restricted further uniformity testing to the original inlet tube configuration.

Because of the high corrosion rates exhibited by samples on the bottom rack, we also eliminated the bottom rack for the final two uniformity tests. Figures 6 and 7 show the weight changes of the samples with exposure time. Again, for clarity, not all curves are labeled with sample numbers. In Figure 6, the curves for samples 2-4 and 7 lie between samples 1 and 8, while in Figure 6, the curves for samples 1, 4, 6, and 8 lie between samples 2 and 3 . These figures show that there is not a deterministic effect of sample placement on weight change data. This is also seen in Tables 4 and 5 which summarize the weight change data. Relative standard deviations for these final two tests are always less than $14 \%$ at each weighing with deviations typically in the $7 \%$ to $8 \%$ range. There is no effect of exposure time on the standard deviations.

We concluded that by using only the top and middle racks for samples, and by using the circular gas inlet tube, the effect of sample placement on corrosion rate would be within experimental error. That is, considering the reproducibility of sample preparation and sample 
weight measurement, a relative standard deviation of less than $14 \%$ (and usually less than $10 \%$ ), based on sample location, is acceptable. 


\section{Reference}

1. W. Abbott, IEEE Trans. Comp. Hybrids Manuficturing Tech., vol. 11, no. 1, (1988). 
Table I. Weight Change Data for the First Uniformity Test

$\begin{array}{cccccc}\text { Sample \# } & \underline{22.5 \mathrm{hr}} & \underline{42.5 \mathrm{hr}} & \underline{113.5 \mathrm{hr}} & \underline{191.5 \mathrm{hr}} & \underline{284 \mathrm{hr}} \\ & & & & & \\ 6 & 20.8 & 38.2 & 77.5 & 102 & 144 \\ 7 & 22.7 & 41.0 & 81.9 & 107 & 149 \\ 8 & 22.4 & 40.4 & 79.1 & 105 & 150 \\ 9 & 24.0 & 43.7 & 90.0 & 120 & 161 \\ 10 & 21.2 & 38.8 & 81.1 & 108 & 147 \\ \text { Average } & 22.2 & 40.4 & 81.9 & 108 & 150 \\ \text { Std. Dev. } & 1.27 & 2.16 & 4.83 & 6.88 & 6.46 \\ \text { Std. Dev. (\%) } & 5.73 & 5.34 & 5.90 & 6.34 & 4.30\end{array}$

Notes: $\quad 1$. Weight changes are in $\mu \mathrm{g} / \mathrm{cm}^{2}$.

2. Std. Dev. (\%) is calculated from (Std. Dev./Average)*10). 
Table II. Weight Change Data for the Second Uniformity Test

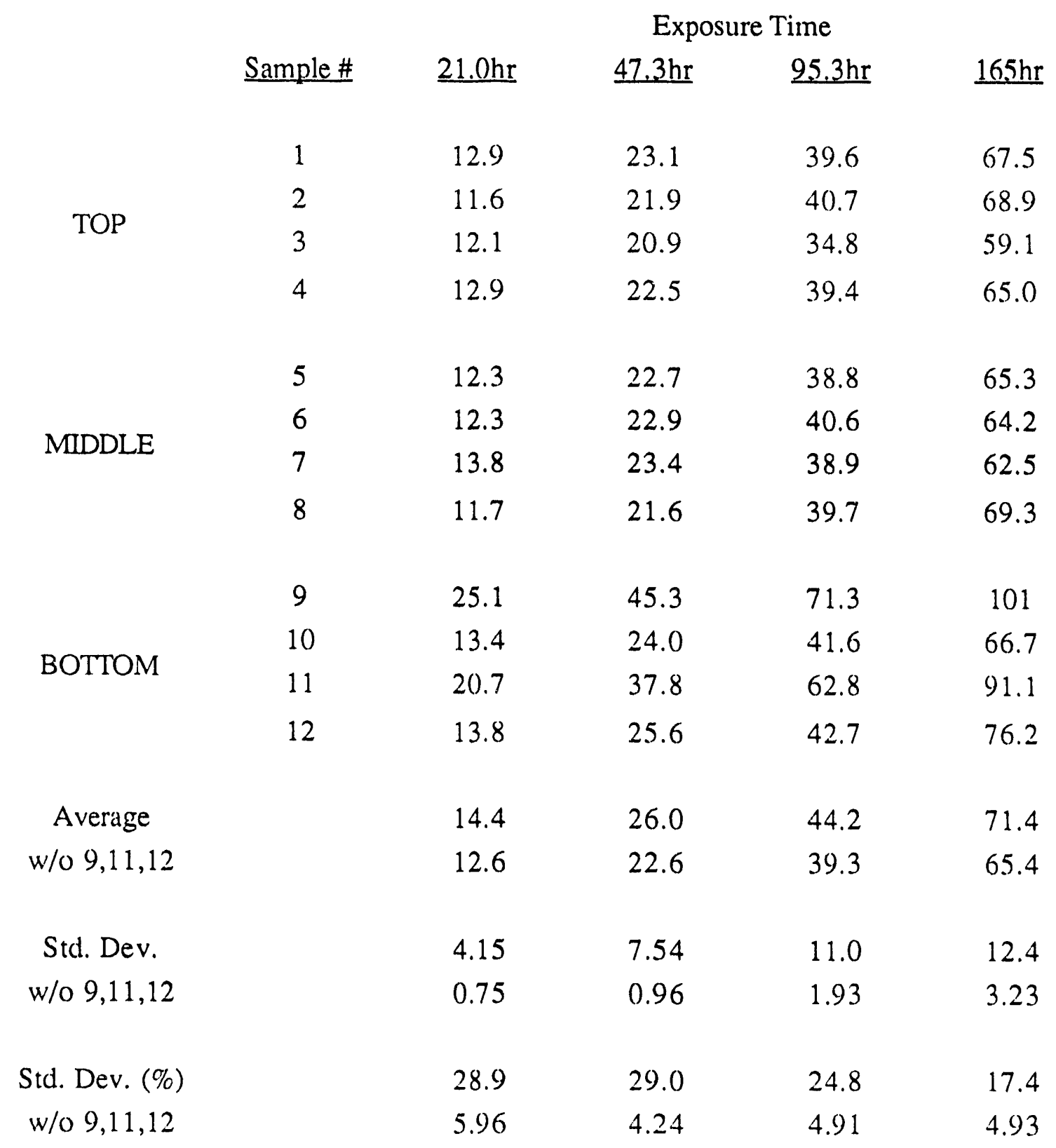

Notes: $\quad$ 1. Weight changes are in $\mu \mathrm{g} / \mathrm{cm}^{2}$.

2. Std. Dev. (\%) is calculated from (Std. Dev./Average)*100. 
Table III. Weight Change Data for the Third Uniformity Test

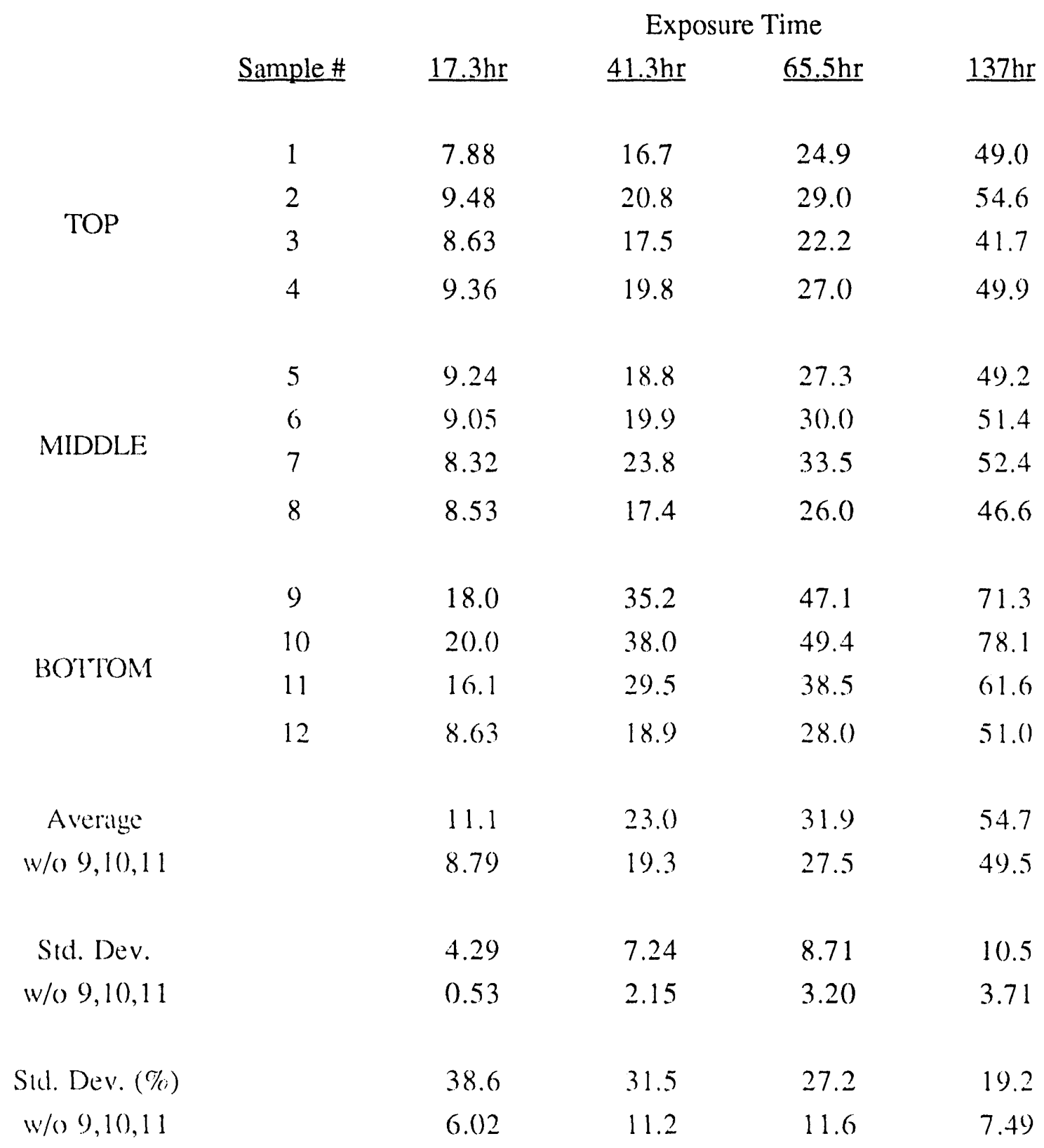

Notes: $\quad$ 1. Weight changes are in $\mu \mathrm{g} / \mathrm{cm}^{2}$.

2. Stcl. Dev. (\%) is calculated from (Stul. Dev./Average)*1()(). 
Table IV. Weight Change Data for the Fourth Uniformity Test

\begin{tabular}{|c|c|c|c|c|c|}
\hline & & & Exp & rime & \\
\hline & $\underline{\text { Sample \# }}$ & 16.0hr & $\underline{40.0 \mathrm{hr}}$ & $\underline{88.0 \mathrm{hr}}$ & $160 \mathrm{hr}$ \\
\hline & 1 & 13.7 & 32.1 & 46.3 & 83.2 \\
\hline גרי דר & 2 & 14.5 & 34.4 & 46.4 & 79.2 \\
\hline IUP & 3 & 15.3 & 30.5 & 42.1 & 79.8 \\
\hline & 4 & 16.8 & 37.4 & 49.7 & 81.7 \\
\hline & 5 & 14.0 & 27.1 & 39.0 & 73.3 \\
\hline$M$ & 6 & 16.7 & 4() .1 & 54.2 & 89.1 \\
\hline IVIIDDLE & 7 & 17.5 & 36.9 & 48.9 & 78.0 \\
\hline & 8 & 13.8 & 29.6 & 41.9 & 77.4 \\
\hline Average & & 15.2 & 33.5 & 46.1 & 80.2 \\
\hline Std. Dev. & & 1.52 & 4.44 & 4.94 & 4.66 \\
\hline d. Dev. $(\%)$ & & 9.95 & 13.3 & 10.7 & 5.81 \\
\hline
\end{tabular}

Notes: $\quad$ 1. Weight changes are in $\mu \mathrm{g} / \mathrm{cm}^{2}$.

2. Std. Dev. (\%) is calculated from (Std. Dev./Average)*10)( 
Table V. Weight Change Data for the Fifth Uniformity Test

\begin{tabular}{|c|c|c|c|c|c|}
\hline & & & Exp & Time & \\
\hline & Sample \# & $18.0 \mathrm{hr}$ & $40.0 \mathrm{hr}$ & $\underline{90.0 \mathrm{hr}}$ & $\underline{184 \mathrm{hr}}$ \\
\hline & 1 & 8.18 & 31.6 & 71.7 & 128 \\
\hline & 2 & 8.69 & 33.1 & 73.1 & 136 \\
\hline TOP & 3 & 7.88 & 30.5 & 65.8 & 121 \\
\hline & 4 & 9.60 & 35.7 & 74.3 & 132 \\
\hline & 5 & 8.87 & 30.7 & 64.9 & 118 \\
\hline & 6 & 9.19 & 35.7 & 78.2 & 134 \\
\hline MIDDLE & 7 & 9.80 & 40.3 & 83.3 & 144 \\
\hline & 8 & 8.18 & 31.2 & 69.1 & 127 \\
\hline Average & & 8.80 & 33.6 & 72.6 & 130 \\
\hline Std. Dev. & & 0.70 & 3.42 & 6.18 & 8.37 \\
\hline td. Dev. $(\%)$ & & 7.94 & 10.2 & 8.52 & 6.44 \\
\hline
\end{tabular}

Notes: $\quad$ 1. Weight changes are in $\mu \mathrm{g} / \mathrm{cm}^{2}$.

2. Std. Dev. (\%) is calculated from (Std. Dev./Average)*100). 


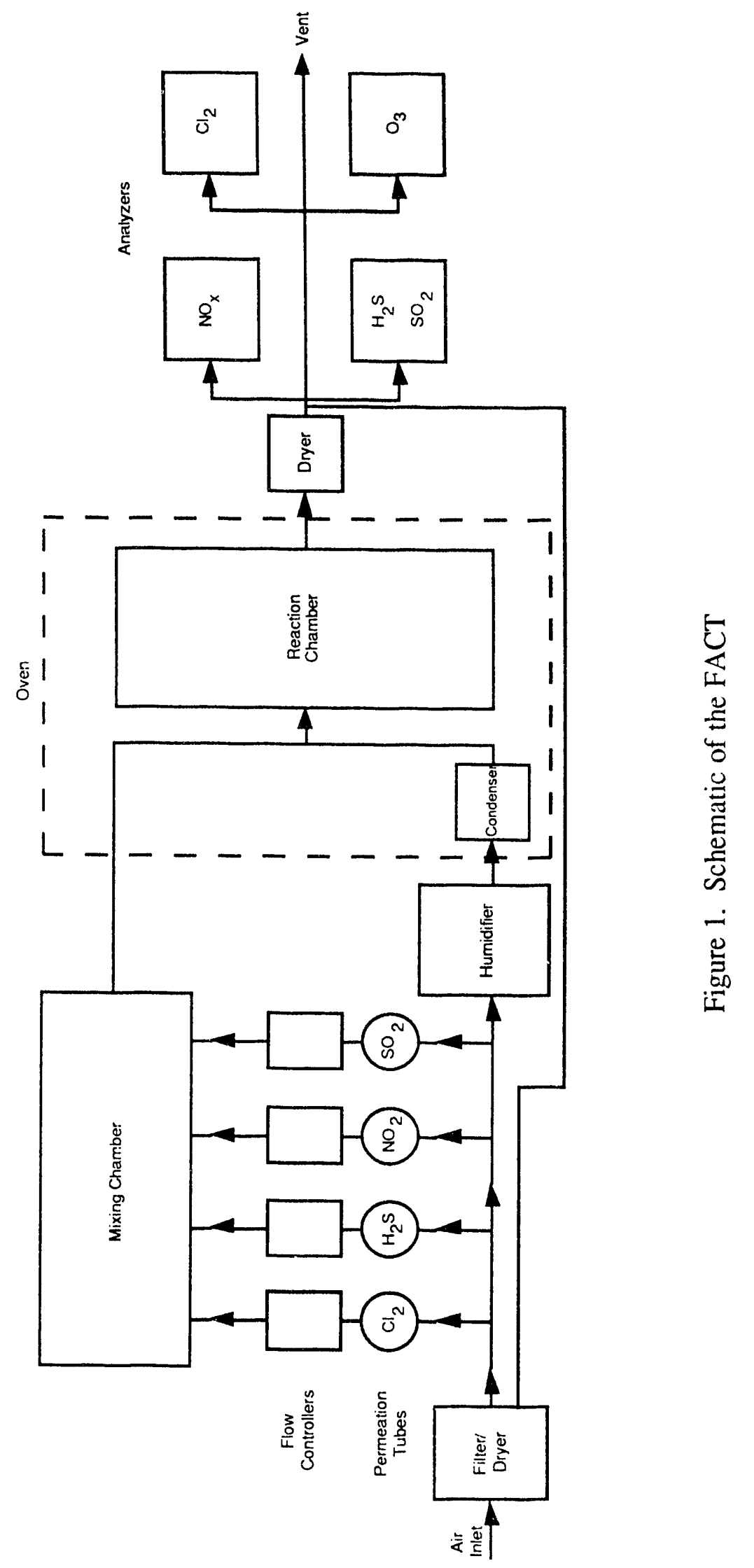




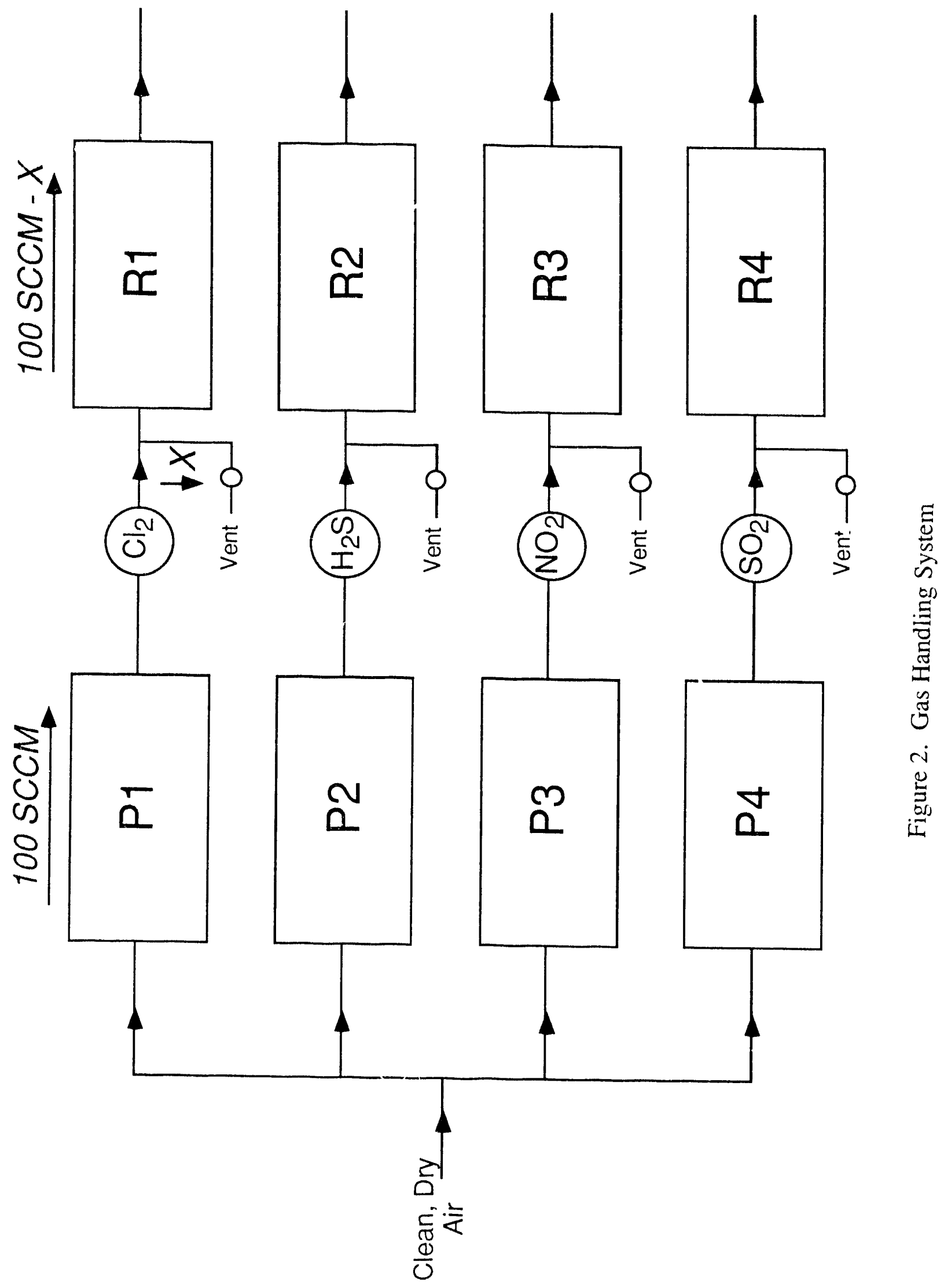




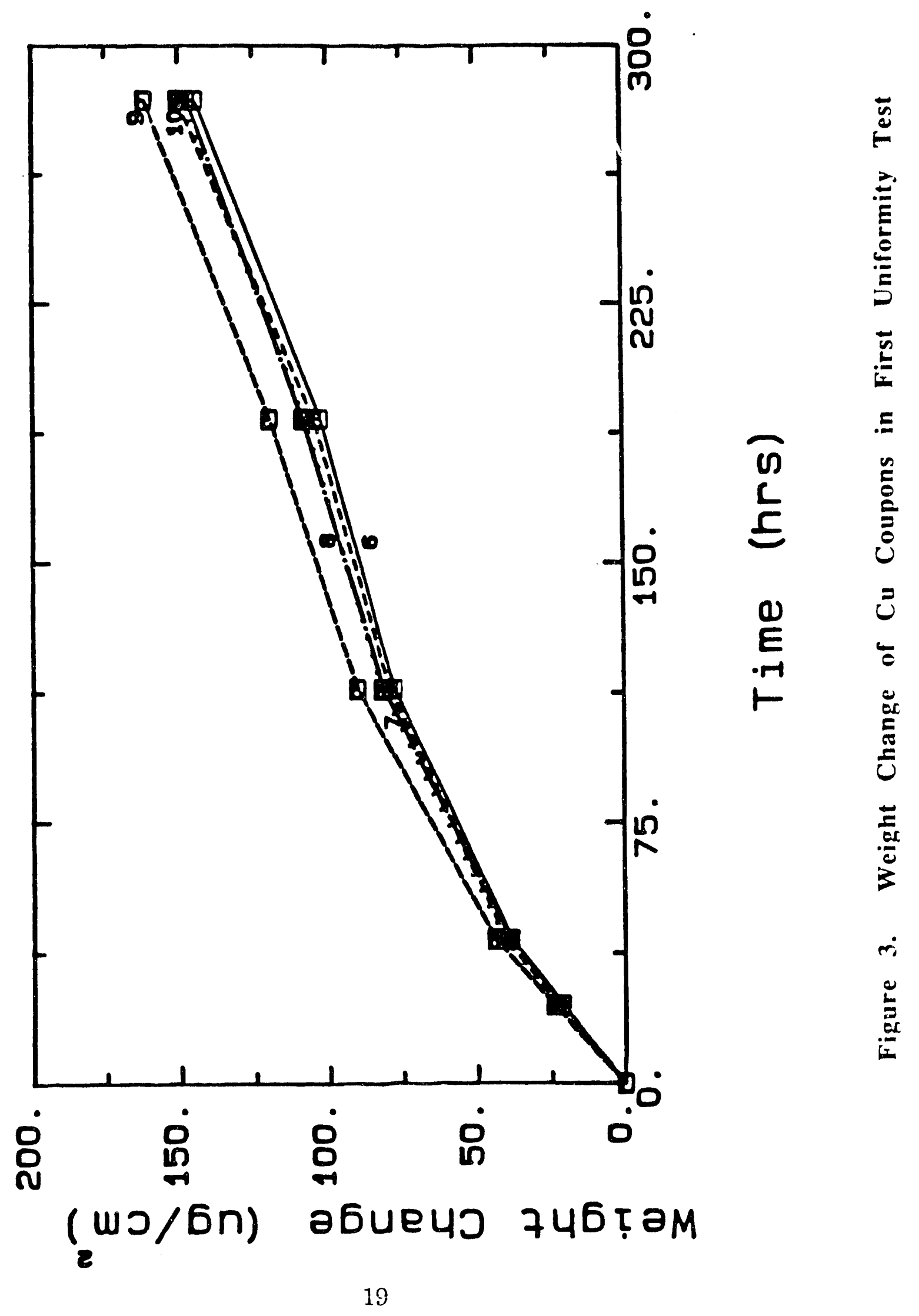




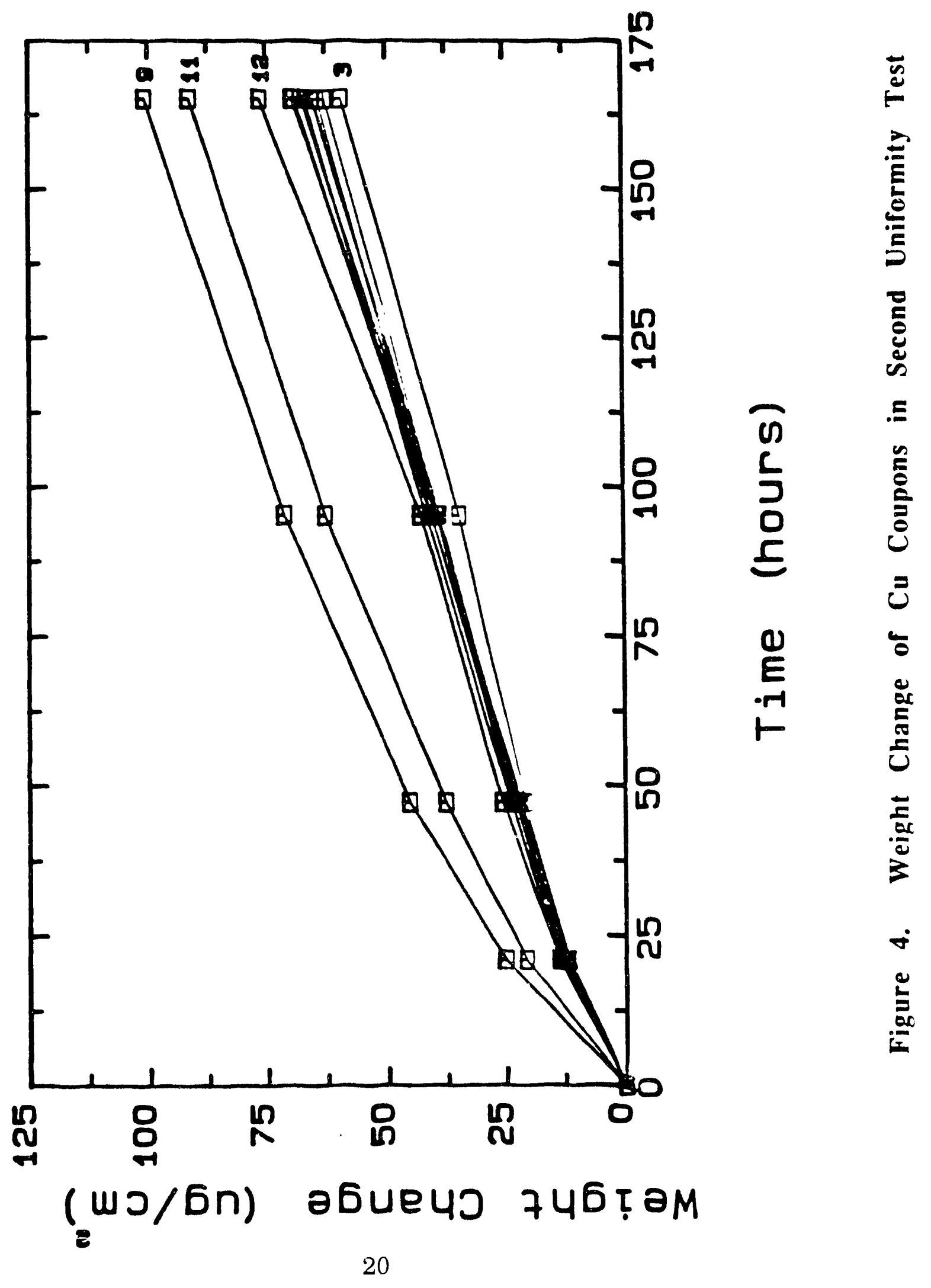




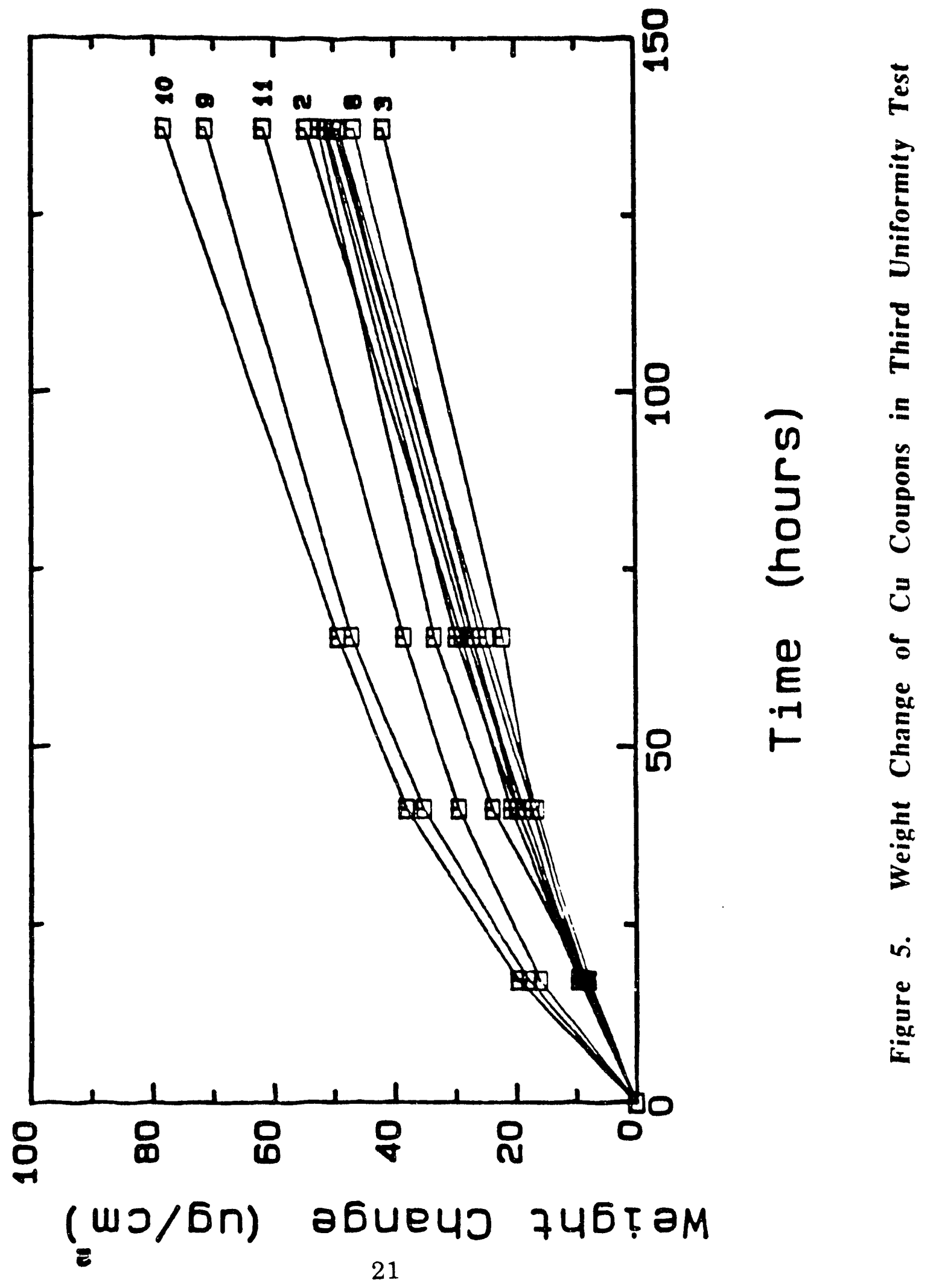




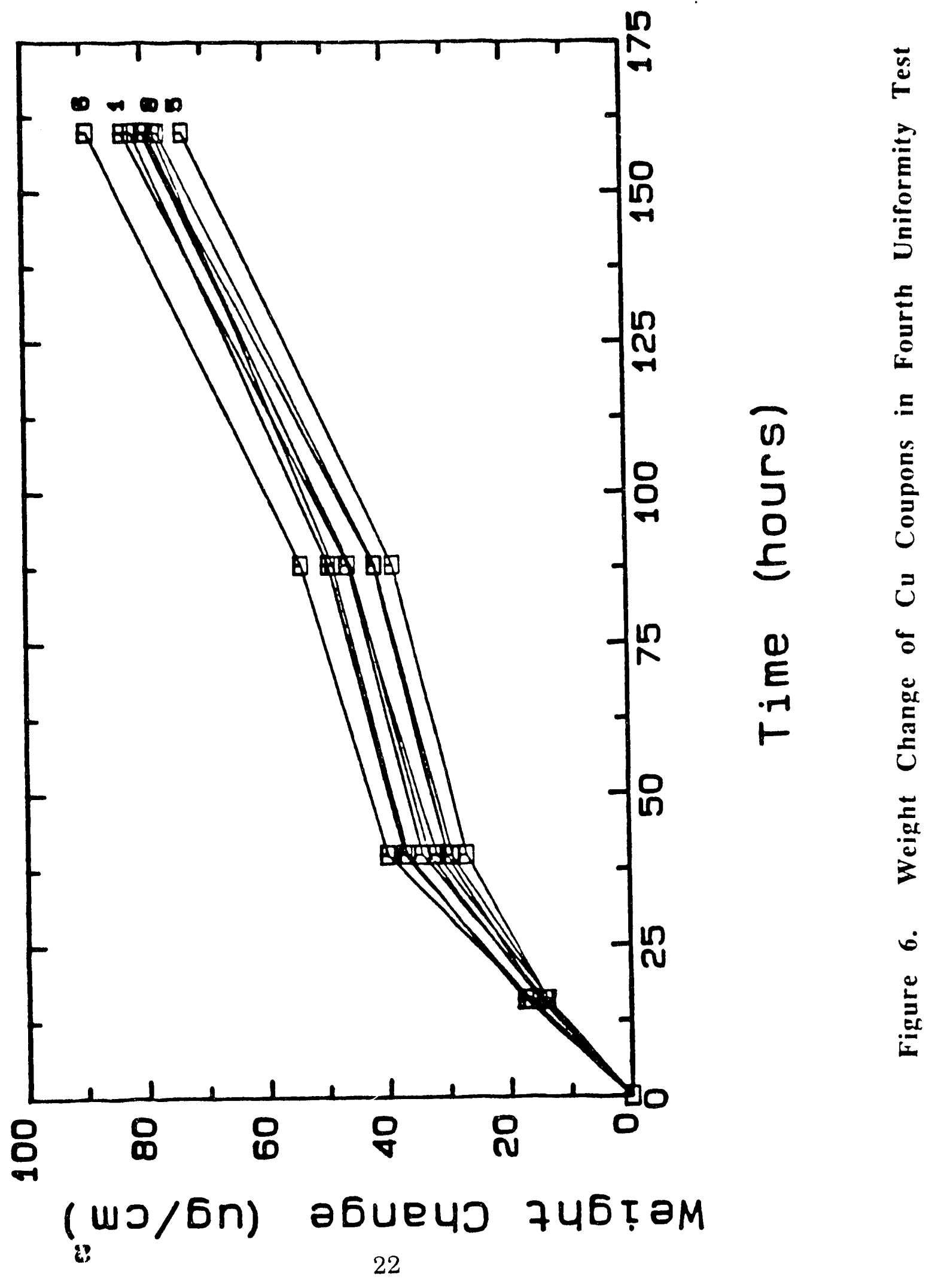




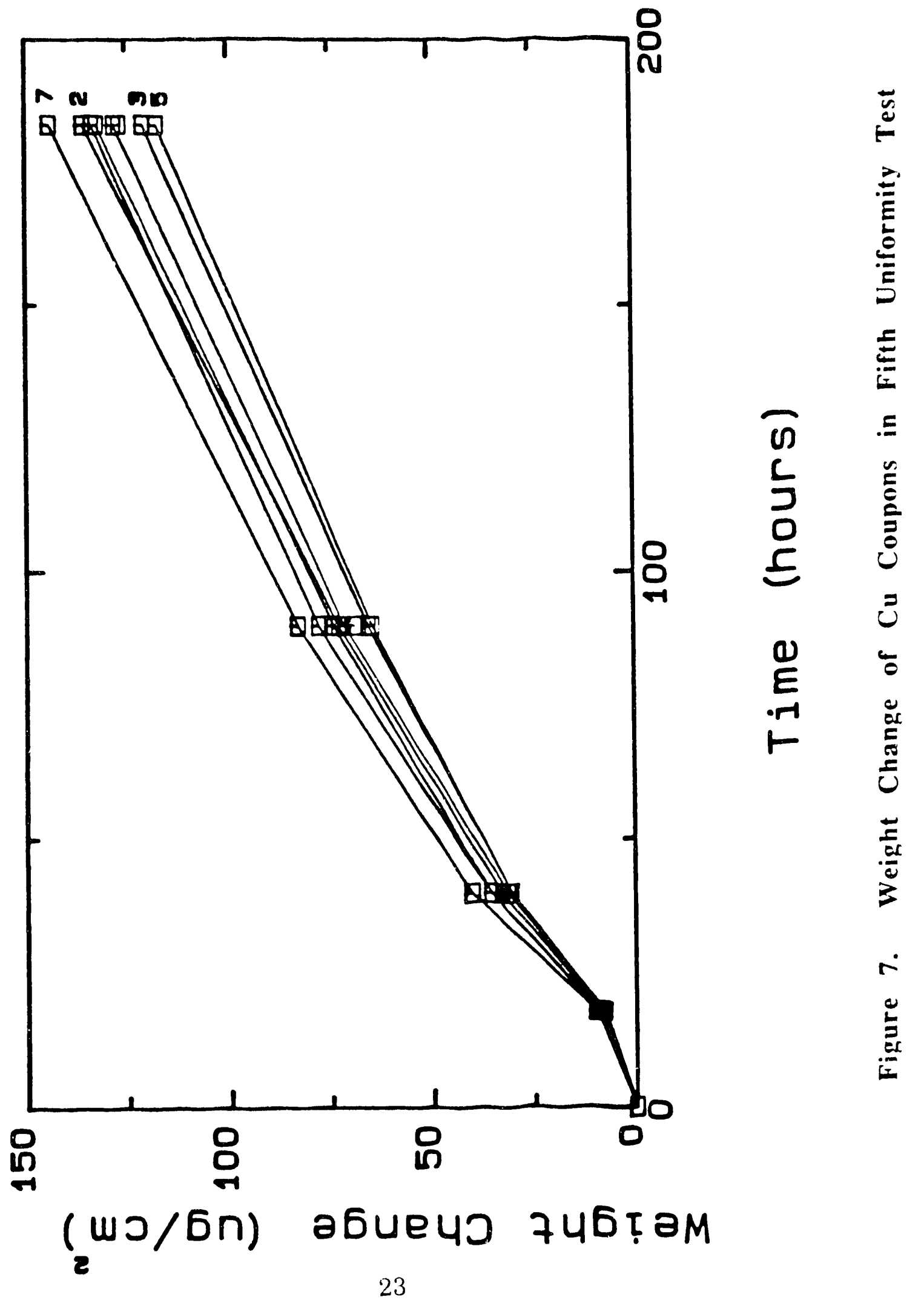


Distribution:
$1800 \quad$ A. D. Romig
1832 R. J. Salzbrenner
1832 R. G. Buchheit
1832 G. A. Poulter (5)
1832 N. R. Sorensen (5)
1841 A. J. Hurd
1841 T. R. Guilinger (5)
1841 J. O. Stevenson
7141 Technical Library (5)
7151 Technical Publications
7613-2 Document Processing for DOE/OSTI (10)
8523-2 Central Technical Files
9611 S. Scott
9611 C. J. Greenholt (5) 


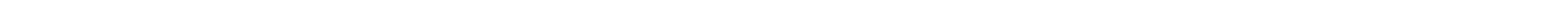




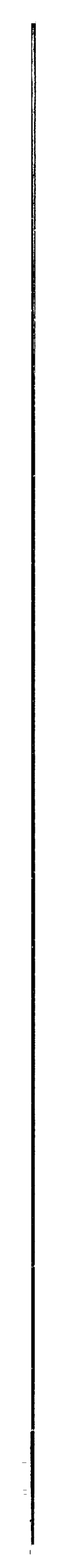

